\title{
Sc-Th (Scandium-Thorium)
}

\section{H. Okamoto}

The Sc-Th phase diagram in [Massalski2] was updated by [1991Oka] based on the work of [1990Ter].

Figure 1 shows the Sc-Th phase diagram calculated by [2009Liu]. The experimental phase boundary data used were essentially taken from [1990Ter]. Accordingly, the diagrams of [1991Oka] and [2009Liu] are similar. The most significant difference is found around the minimum melting point. In the phase diagram of [19910ka], the liquidus curve showed sudden curvature change, which is unlikely. The shape shown in Fig. 1 seems to be more normal.

Table 1 shows Sc-Th crystal structure data.

\section{References}

1990Ter: G.I. Terekhov and S.I. Sinyakova, The Th-Sc Phase Diagram, Izv. Akad. Nauk SSSR, Met., 1990, (3), p 215-218, in Russian

19910ka: H. Okamoto, Sc-Th (Scandium-Thorium), J. Phase Equilib., 1991, 12(1), p 123

2009Liu: X.J. Liu, S.L. Wang, and C.P. Wang, "Thermodynamic Assessments of the Sc-M (M: Ag, B and Th)" Systems, J. Alloys Compd., 2009, 469, p 186-192

Table 1 Sc-Th crystal structure data

\begin{tabular}{|c|c|c|c|c|c|}
\hline Phase & Composition, at.\% Th & Pearson symbol & Space group & Strukturbericht designation & Prototype \\
\hline$(\beta \mathrm{Sc}, \beta \mathrm{Th})$ & $0-100$ & $c I 2$ & $\operatorname{Im} \overline{3} m$ & $A 2$ & $\mathrm{~W}$ \\
\hline$(\alpha \mathrm{Sc})$ & $0-21.2$ & $h P 2$ & $P 6_{3} / m m c$ & $A 3$ & $\mathrm{Mg}$ \\
\hline$(\alpha \mathrm{Th})$ & $36.7-100$ & $c F 4$ & $F m \overline{3} m$ & $A 1$ & $\mathrm{Cu}$ \\
\hline
\end{tabular}

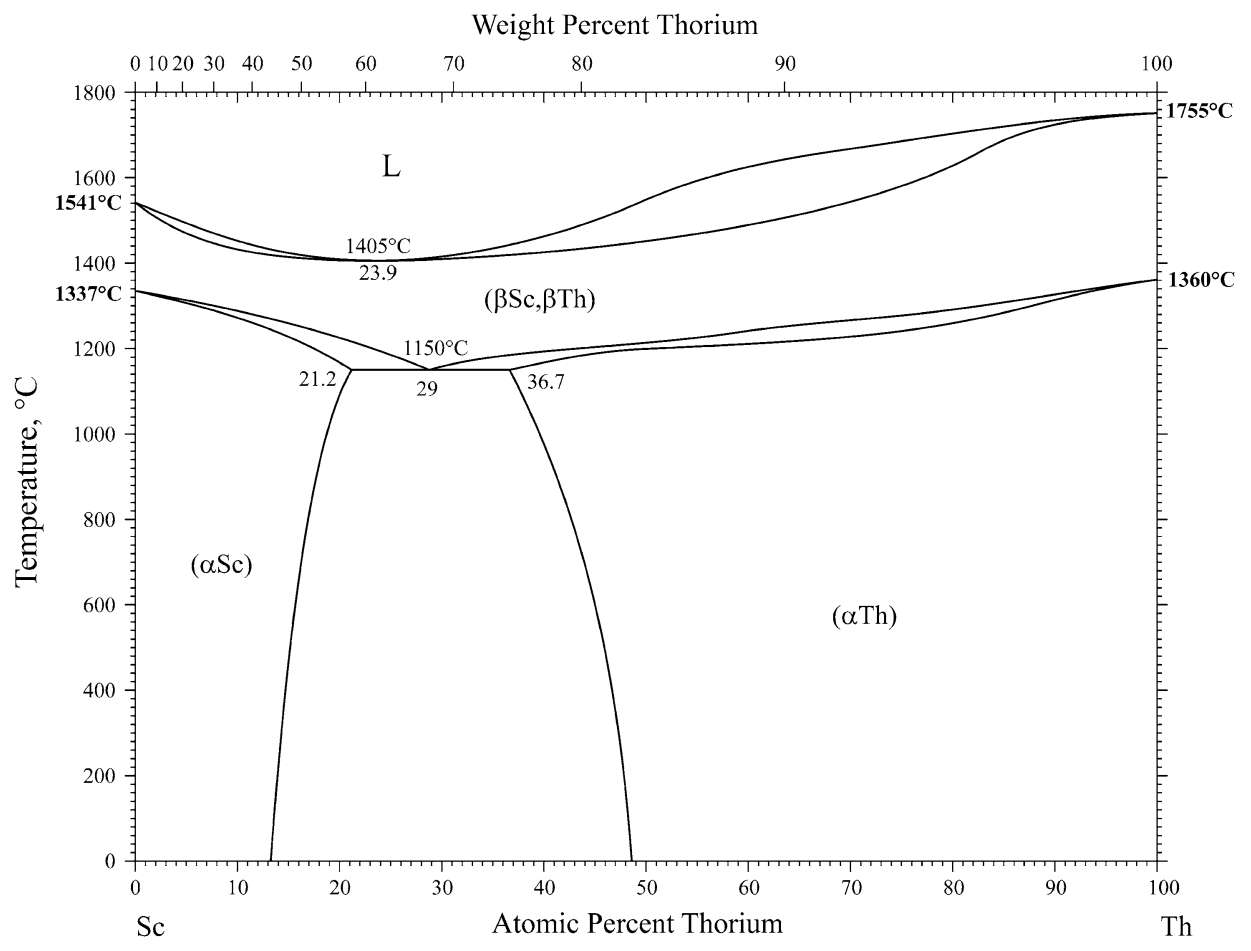

Fig. 1 Sc-Th phase diagram 